\title{
Erratum to: The capacitated max $k$-cut problem
}

\author{
Daya Ram Gaur • Ramesh Krishnamurti • \\ Rajeev Kohli
}

\section{Erratum to: Math. Program., Ser. A (2008) 115:65-72 DOI 10.1007/s10107-007-0139-z}

The algorithm described by the authors in a recent issue of this journal (The capacitated max $k$-cut problem, 115(1): 65-72 (2008)) is not guaranteed to terminate. To see this, consider a complete graph over $n$ vertices, identical weights on all edges, where a bipartition is desired with capacities of 1 and $n-1$ (brought to our attention by Prof. Jaikumar Radhakrishnan). The lower bound for general $k$ approaches the lower bound obtained for $k=2$ by considering the case where the capacity of all but two sets is 0 (kindly brought to our attention by Dr. Maxim Sviridenko). Thus, it is unlikely a better lower bound (which improves with increasing $k$ ) can be obtained.

The online version of the original article can be found under doi:10.1007/s10107-007-0139-z.

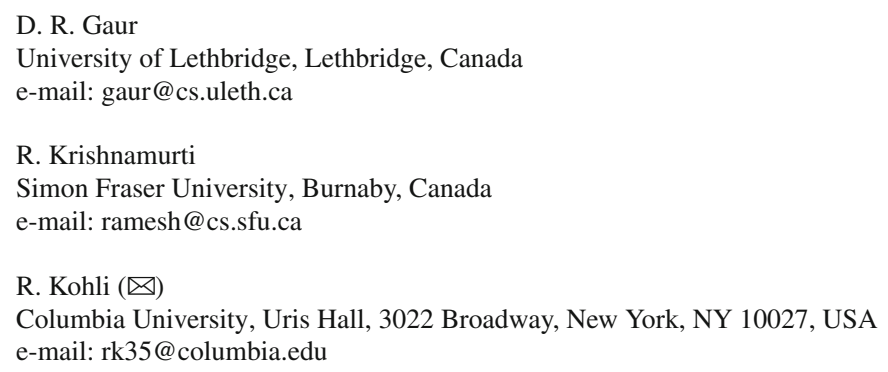

